\title{
TEMPERATURE DISTRIBUTIONS IN ROTATING FLOWS NORMAL TO A FLAT SURFACE*
}

\author{
BY \\ HANS J. LUGT AND ERNST W. SCHWIDERSKI \\ U. S. Naval Weapons Laboratory, Dahlgren, Va.
}

\begin{abstract}
The temperature fields of rotating laminar flows over a flat surface are investigated. Dissipation is considered, whereas free convection effects are neglected. The calculation is based on the knowledge of the flow fields, which have been studied in previous papers by the authors. In these papers first order approximations reduce the Navier-Stokes equations to ordinary differential equations. The basic ideas of this method are applied to the energy equation. In the special case of negligible dissipation the heat transfer of von Kármán type flows can be compared with existing experimental data. The agreement is satisfactory.

1. Introduction. The solution of a convective heat transfer problem is closely connected with the knowledge of the associated fluid motion since both the temperature distribution and the velocity field interact mutually. This dependence is only unilateral for an incompressible fluid with constant viscosity because the temperature distribution does not influence the flow field. For this reason solutions of the equations of motion, which are known from other investigations, can be used in order to obtain solutions of the associated heat transfer problems.
\end{abstract}

In recent papers $[1,2]$ the authors have studied rotating flows over a flat surface by means of local first order approximations of the Navier-Stokes equations. Numerical results have been computed for the vortex flow normal to a flat surface, for the rotating disk in a fluid at rest (von Kármán problem), and for the solid-body rotation of a fluid over a fixed flat plate (Bödewadt problem).

In this paper the basic ideas of the new method are applied to the temperature fields of the above mentioned flows. Numerical solutions are presented for all problems.

2. Basic equations. For a steady, axisymmetric, and laminar flow the NavierStokes equations, the equation of continuity, and the energy equation, in cylindrical coordinates $(r, \varphi, z)$, are

$$
\begin{aligned}
u u_{r}+w u_{z}-\frac{v^{2}}{r} & =-\frac{1}{\rho} p_{r}+\nu\left[u_{r r}+\left(\frac{u}{r}\right)_{r}+u_{z z}\right], \\
u v_{r}+w v_{z}+\frac{w v}{r} & =+\nu\left[v_{r r}+\left(\frac{v}{r}\right)_{r}+v_{z z}\right], \\
u w_{r}+w w_{z} & =-\frac{1}{\rho} p_{z}+\nu\left[w_{r r}+\frac{1}{r} w_{r}+w_{z z}\right], \\
(r u)_{r}+(r w)_{z} & =0,
\end{aligned}
$$

${ }^{*}$ Received March 17, 1964; revised manuscript received September 1, 1964. Part of this paper was presented at the Meeting of the American Physical Society, Division of Fluid Dynamics, at MIT, Cambridge, Mass., 25-27 November, 1963. 


$$
\begin{aligned}
& c\left(u T_{r}+w T_{z}\right)=\frac{k}{\rho}\left(T_{r r}+\frac{1}{r} T_{r}+T_{z z}\right) \\
& +\nu\left[2 u_{r}^{2}+2\left(\frac{u}{r}\right)^{2}+2 w_{z}^{2}+\left(v_{r}-\frac{v}{r}\right)^{2}+\left(u_{z}+w_{r}\right)^{2}+v_{z}^{2}\right] .
\end{aligned}
$$

The quantities $u, v, w$ denote the velocity components, $T$ the temperature, and $p$ the pressure of the fluid. The constants $\rho, \nu, c, k$ are the density, the kinematic viscosity, the specific heat, and the conductivity, respectively. Free convection phenomena are not considered. The boundary conditions for Eqs. (1) through (5) depend on the special problems under consideration. They will be discussed in the following two sections together with a reduction of the corresponding energy equations to ordinary differential equations.

3. Temperature fields of vortex flows. The axisymmetric vortex flow over a flat surface produced by an infinitely long rotating rod has been examined in [1]. Proper boundary conditions have been obtained in close connection with the description of the flow model. In an analogous way the corresponding boundary data for the energy equation (5) can be determined if the temperature field far away from the surface is known. Using the flow field outside the boundary layer (see [1])

$$
u \equiv 0, \quad v=\frac{\Gamma}{r}, \quad w=\Gamma A \log \frac{r_{0}}{r},
$$

and assuming the restriction $T=T(r)$ one arrives at the simplified energy equation

$$
T_{r r}+\frac{T_{r}}{r}=-\frac{\mu}{k} \Gamma^{2}\left(\frac{4}{r^{4}}+\frac{A^{2}}{r^{2}}\right) .
$$

The constant $\mu=\nu \rho$ is the dynamic viscosity, $\Gamma$ represents the circulation of the vortex outside the boundary layer, and $A$ is a measure for the strength of the source-sink pair

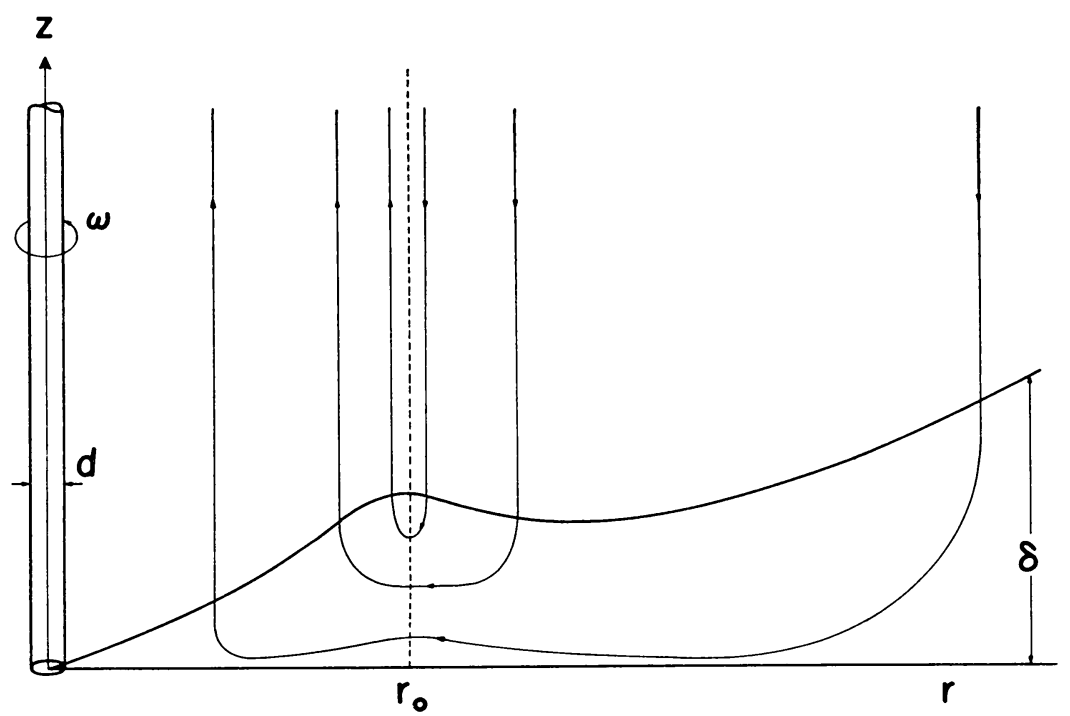

FIG. 1. Scheme of the secondary flow produced by a vortex motion normal to a flat surface. 
at $z=\infty$. The line $r=r_{0}$ marks the change between the sink and the source (see Fig. 1). Eq. (7) yields the general solution

$$
T=t_{0}+\frac{\mu \Gamma^{2}}{k}\left(\frac{1}{r_{0}}-\frac{1}{r}\right)+t_{1} \log \frac{r_{0}}{r}-\frac{\mu \Gamma^{2} A^{2}}{2 k} \log ^{2} \frac{r_{0}}{r}=\tau(r)
$$

with $t_{0}$ and $t_{1}$ as integration constants. The parameter $t_{0}$ is chosen in such a way that it represents the temperature difference between the surface and the flow outside the boundary layer at $r=r_{0}$. The constant $t_{1}$ is a measure for the strength of a heat source which may be permitted in the core (see Sec. 5). Both $t_{0}$ and $t_{1}$ are determined by Eq. (8) if the measured temperatures of any two appropriate points $\left(r_{1}, z_{1}\right)$ and $\left(r_{2}, z_{2}\right)$ of the flow field are known.

Assuming an isothermal surface one can specify the boundary conditions for the energy equation (5). They are together with the boundary data for equations (1) through (4) (see [1]):

$$
\begin{aligned}
& \left.\begin{array}{l}
r>0, z=0 \\
r=\infty, z<\infty
\end{array}\right\}: u=0, \quad v=0, \quad w=0, \quad T=T_{w}=\text { const }, \\
& \left.\begin{array}{l}
r \rightarrow 0, z>0 \\
r<\infty, z \rightarrow \infty
\end{array}\right\}: u \rightarrow 0, \quad \frac{r v}{\Gamma} \rightarrow 1, \quad \frac{w}{\Gamma A \log \frac{r_{0}}{r}} \rightarrow 1, \quad \frac{T-T_{w}}{\tau(r)} \rightarrow 1 .
\end{aligned}
$$

No conditions are prescribed at the singular points $(r=0, z=0)$ and $(r=\infty, z=\infty)$. The introduction of the "boundary layer transformation"

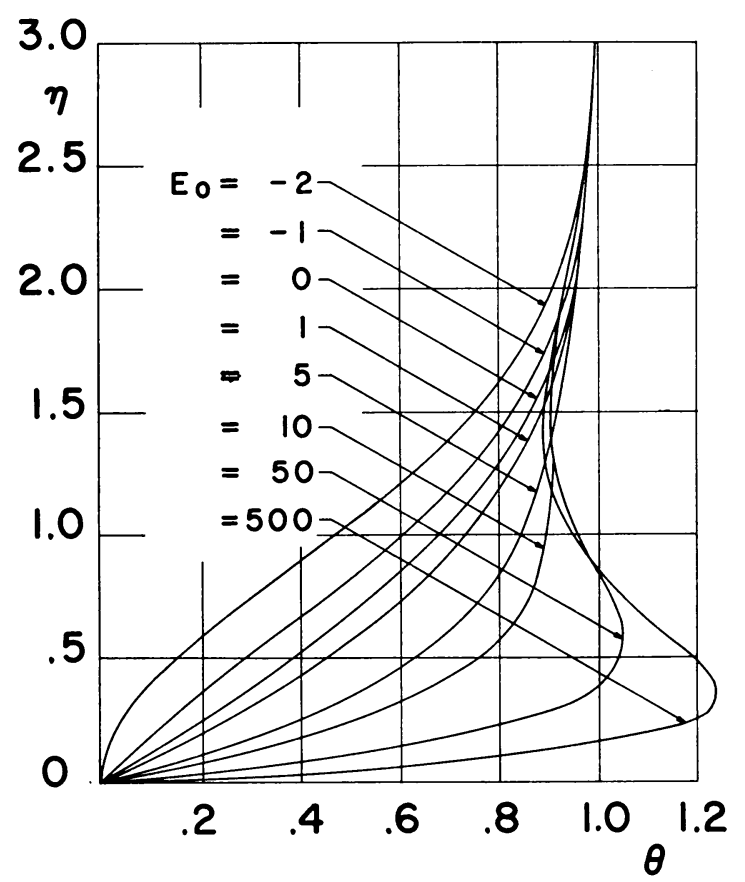

FIg. 2. The dimensionless temperature $\theta$ of vortex flows vs. the dimensionless variable $\eta$ for $R=1, P_{r}=.7,1 / E_{1}=0$, and for different $E_{0}$. 


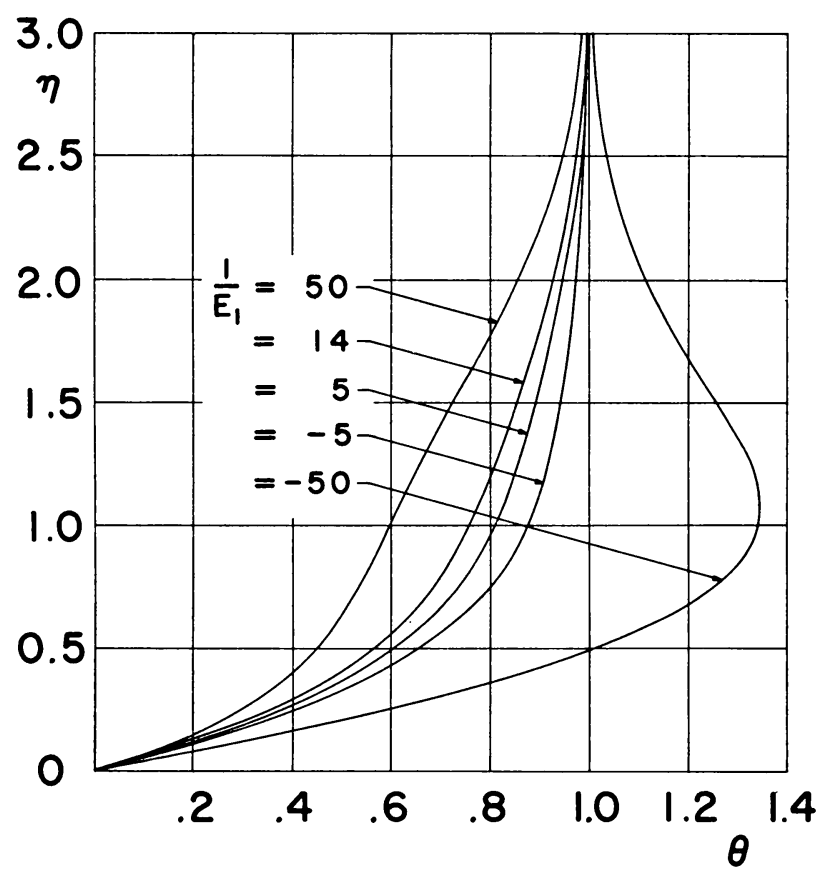

Fia. 3. The dimensionless temperature $\theta$ of vortex flows vs. the dimensionless variable $\eta$ for $R=1, P_{r}=.7, E_{0}=5$, and for different $E_{1}$.

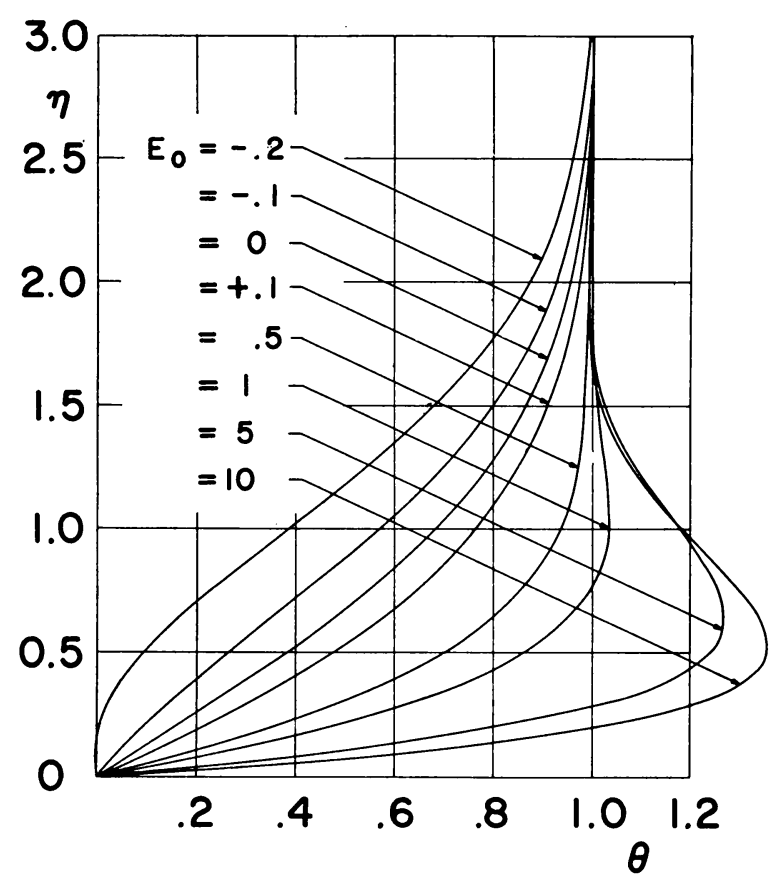

Fig. 4. The dimensionless temperature $\theta$ of vortex flows vs. the dimensionless variable $\eta$ for $R=1, P_{r}=7,1 / E_{1}=0$, and for different $E_{0}$. 


$$
r=r, \quad \eta=\frac{z}{\gamma(r)}
$$

where $z=\sigma \gamma(r)$ with a proper constant $\sigma$ (see [1]) is the unknown "limiting line of the boundary layer" along the surface at $z=0$, led to the "adjustable local boundary layer approximation of first order" around $r=r_{0}$

$$
\begin{gathered}
u=-\frac{\Gamma}{r} g^{\prime}(\eta), \quad v=\frac{\Gamma}{r} V(\eta), \quad w=\frac{2 \Gamma}{r_{0}} \log \frac{r_{0}}{r}\left[g(\eta)-\eta g^{\prime}(\eta)\right], \\
\underline{\rho}=-\frac{\Gamma}{2 r^{2}} P(\eta), \quad A=\frac{2}{r_{0}} g_{\infty}, \quad R=\frac{\Gamma}{\nu} .
\end{gathered}
$$

The variable $g$ is the dimensionless stream function and $R$ is the Reynolds number. The primes denote derivatives with respect to $\eta$. The corresponding approximation for the temperature $T$ is

$$
T=\tau(r) \theta(\eta)+T_{w}
$$

In connection with the dimensionless quantities (12) the approximation (13) reduces the energy equation to

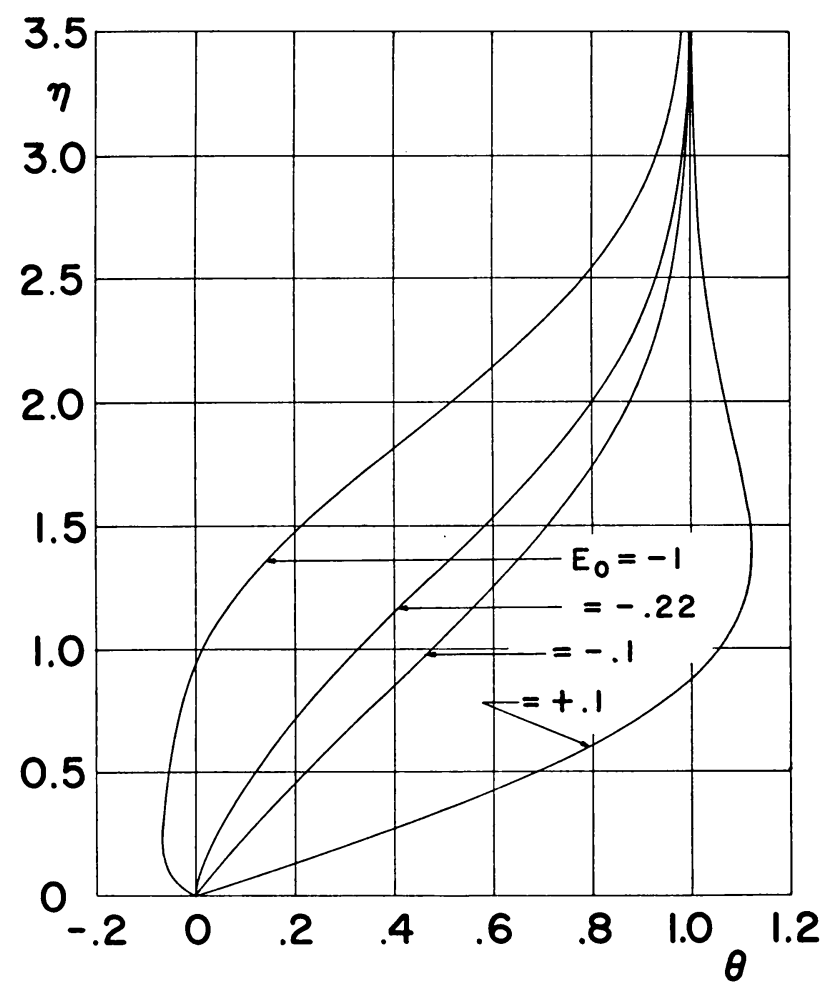

FIG. 5. The dimensionless temperature $\theta$ of vortex flows vs. the dimensionless variable $\eta$ for $R=100, P_{r}=.7,1 / E_{1}=0$, and for different $E_{0}$. 


$$
\begin{aligned}
{[1} & \left.+\left(\frac{d \gamma}{d r}\right)^{2} \eta^{2}\right] \theta^{\prime \prime}+\left[\eta\left\{2\left(\frac{d \gamma}{d r}\right)^{2}-\gamma \frac{d^{2} \gamma}{d r^{2}}-\left(1+\frac{2 r}{\tau} \frac{d \tau}{d r}\right) \frac{\gamma}{r} \frac{d \gamma}{d r}\right\}-R P_{r} \frac{\gamma}{r}\left\{\eta g^{\prime} \frac{d \gamma}{d r}\right.\right. \\
& \left.\left.+2 \frac{r_{0}}{r} \log \frac{r_{0}}{r}\left(g-\eta g^{\prime}\right)\right\}\right] \theta^{\prime}+\frac{\gamma^{2}}{\tau}\left[\frac{d^{2} \tau}{d r^{2}}+\frac{1}{r}+\frac{R}{r} P_{r} g^{\prime} \frac{d \tau}{d r}\right] \theta \\
& =\frac{\gamma^{2} \Gamma^{2}}{r^{2} c \tau} P_{r}\left[2\left(\frac{\eta}{\gamma} g^{\prime \prime} \frac{d \gamma}{d r}+\frac{g^{\prime}}{r}\right)^{2}+\frac{2}{r^{2}}{g^{\prime}}^{2}+8\left(\frac{r_{0} r}{\gamma} \eta g^{\prime \prime} \log \frac{r_{0}}{r}\right)^{2}\right. \\
& \left.+\left(\frac{\eta}{\gamma} V^{\prime} \frac{d \gamma}{d r}+2 \frac{V}{r}\right)^{2}+\left\{\frac{g^{\prime \prime}}{\gamma}+\frac{2}{r_{0}}\left(g-\eta g^{\prime}\right)-2 \frac{r \eta^{2}}{r_{0} \gamma} g^{\prime \prime} \frac{d \gamma}{d r} \log \frac{r_{0}}{r}\right\}^{2}+\frac{V^{\prime 2}}{\gamma^{2}}\right],
\end{aligned}
$$

where the dimensionless parameter $P_{r}=c \mu / k$ is the Prandtl number of the fluid.

Simultaneously with the reduction of the Navier-Stokes equations it was found in [1] for the function $\gamma$ near $r=r_{0}$

$$
\gamma(r)=\frac{r_{0}}{2}\left(\frac{r}{r_{0}}\right)^{2}\left(1+2 \log \frac{r_{0}}{r}\right)+\cdots .
$$

For $r=r_{0}$ Eq. (14) reduces to

$$
\begin{aligned}
\theta^{\prime \prime}+\eta \theta^{\prime}-P_{r} E_{0}\left[1+g_{\infty}^{2}\right. & \left.+\frac{R}{4}\left(\frac{1}{E_{1}}-2 P_{r}\right) g^{\prime}\right] \theta \\
& =-E_{0} P_{r}\left[g^{\prime 2}+V^{\prime 2}+V^{2}+\left(g^{\prime \prime}+g-\eta g^{\prime}\right)^{2}\right],
\end{aligned}
$$

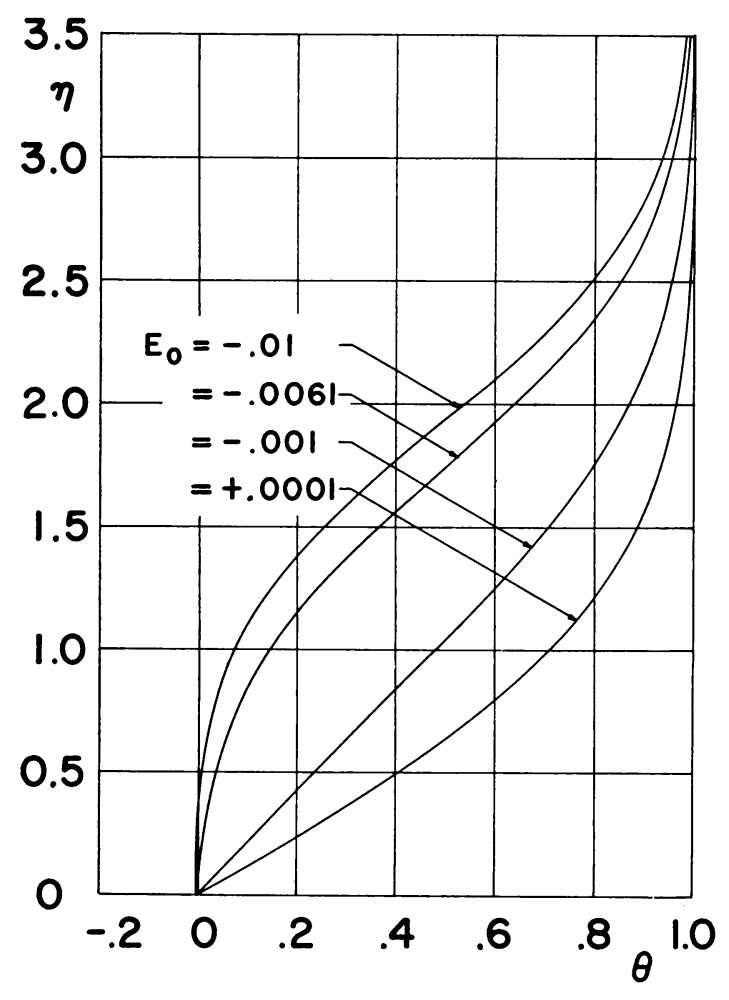

Fig. 6. The dimensionless temperature $\theta$ of vortex flows vs. the dimensionless variable $\eta$ for $R=100, P_{r}=7,1 / E_{1}=0$, and for different $E_{0}$. 
if one interprets the constants

$$
E_{0}=\frac{\Gamma^{2}}{c t_{0} r_{0}^{2}}, \quad E_{1}=\frac{\Gamma^{2}}{c t_{1} r_{0}^{2}}
$$

as Eckert numbers of the combined flow and temperature fields. The boundary conditions are

$$
\begin{aligned}
\eta=0: & \theta=0, \\
\eta=\infty: & \theta=1 .
\end{aligned}
$$

As in [1] it is useful to reduce the ordinary boundary value problem to a Volterra integral equation. Defining the auxiliary function

$D(\eta)=P_{r} E_{0}\left\{\left[1+g_{\infty}^{2}+\frac{R}{4}\left(\frac{1}{E_{1}}-2 P_{r}\right) g^{\prime}\right] \theta-g^{\prime 2}-V^{\prime 2}-V^{2}-\left(g^{\prime \prime}+g-\eta g^{\prime}\right)^{2}\right\}$,

one obtains the linear equation

$$
\theta^{\prime \prime}+\eta \theta^{\prime}=D(\eta)
$$

With the aid of the error function

$$
V=\operatorname{erf}\left(2^{-1 / 2} \eta\right)=2 \pi^{-1 / 2} \int_{0}^{\eta / 2^{1 / 2}} e^{-t^{2}} d t
$$

Table 1: Nusselt Numbers of Vortex Flows

1. $R=1, P_{r}=0.7,1 / E_{1}=0$.

\begin{tabular}{l|rrrrrrrr}
$E_{0}$ & -2 & -1 & 0 & +1 & 5 & 10 & 50 & 500 \\
\hline$N$ & -.004 & +.644 & 1.13 & 1.53 & 2.74 & 3.85 & 9.16 & 39.6
\end{tabular}

2. $R=1, P_{r}=0.7, \quad E_{0}=5$.

\begin{tabular}{l|rrrrrrr}
$1 / E_{1}$ & -50 & -10 & -5 & 0 & +5 & 14 & 50 \\
\hline$N$ & 3.92 & 2.91 & 2.82 & 2.74 & 2.67 & 2.55 & 2.19
\end{tabular}

3. $R=1, P_{r}=7,1 / E_{1}=0$.

\begin{tabular}{l|rrrrrrr}
$E_{0}$ & -.2 & -.1 & 0 & +.1 & 1 & 5 & 10 \\
\hline$N$ & -.173 & +.569 & 1.13 & 1.59 & 4.17 & 9.85 & 13.61
\end{tabular}

4. $R=100, P_{r}=0.7,1 / E_{1}=0$.

\begin{tabular}{l|rrrrr}
$E_{0}$ & -1 & -.22 & -.1 & 0 & +.1 \\
\hline$N$ & -1.47 & +.002 & .490 & 1.13 & 2.28
\end{tabular}

5. $R=100, P_{r}=7,1 / E_{1}=0$.

\begin{tabular}{l|rrrrr}
$E_{0}$ & -.01 & -.0061 & -.001 & 0 & +.0001 \\
\hline$N$ & -.116 & .000 & +.647 & 1.13 & 1.20
\end{tabular}


the general solution of Eq. (21) can be written in the form

$$
\theta(\eta)=\lambda V(\eta)+\lambda^{*}+\int_{0}^{\eta} \frac{D(t)}{V^{\prime}(t)}[V(\eta)-V(t)] d t .
$$

With the boundary conditions (18) and (19) one arrives at the Volterra integral equation

$$
\theta(\eta)=\lambda V(\eta)+\int_{0}^{\eta} \frac{D(t)}{V^{\prime}(t)}[V(\eta)-V(t)] d t,
$$

where

$$
\lambda=1-\int_{0}^{\infty} \frac{D(t)}{V^{\prime}(t)}[1-V(t)] d t .
$$

The nonlinear Volterra integral equation (24) can be solved by a successive approximation which may be initiated with $\theta_{0}=V$. Numerical results are displayed in Sect. 5 .

4. Temperature fields of von Kármán-Bödewadt flows. The heat transfer from a rotating disk was investigated by Wagner [3], Millsaps and Pohlhausen [4], and Sparrow and Gregg [5], who used von Kármán's solution of the equations of motion [6]. Young [7],

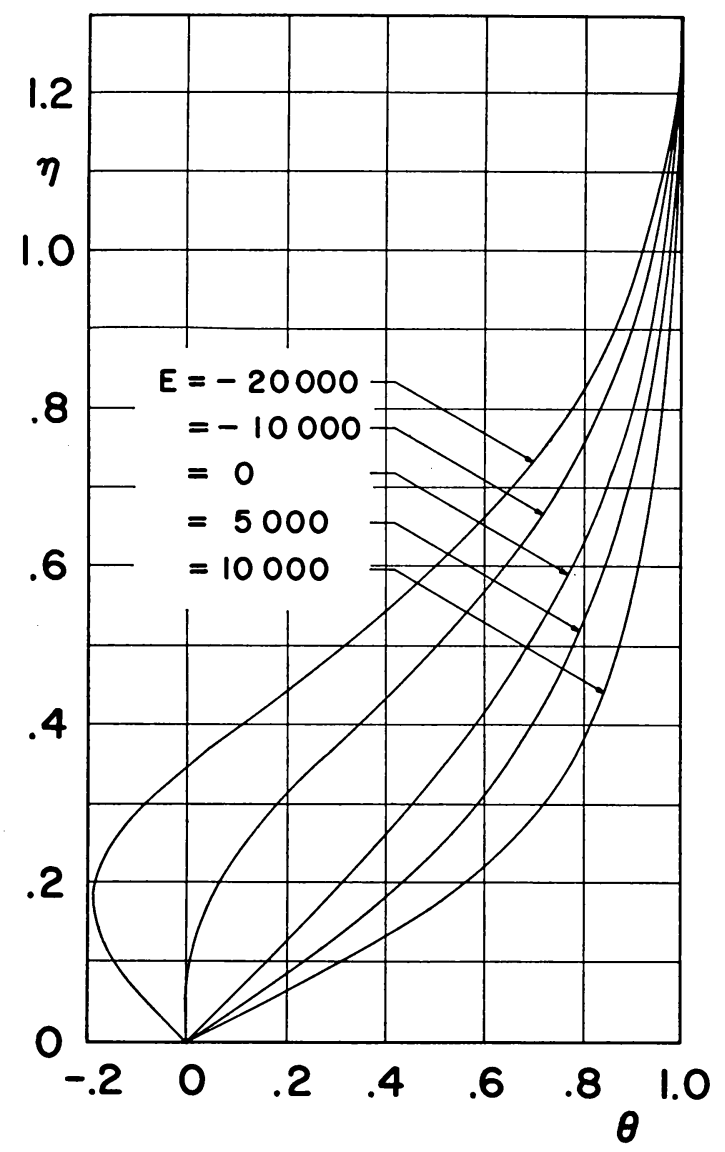

FIG. 7. The dimensionless temperature $\theta$ of Kármán flows vs. the dimensionless variable $\eta$ for $R=1, P_{r}=.7$, and for different $E$. 
Cobb and Saunders [8] studied the problem experimentally. In this section the derivations are based on the flow field which is computed in [2]. Simultaneously, the heat transfer from a fixed disk by a solid-body rotation of a fluid (Bödewadt problem) is examined.

Similar to the explanations of the previous section the boundary conditions for the energy equation are determined by the temperature field outside the boundary layer. The flow fields at that region are described by

$$
u \equiv 0, \quad v=\kappa \omega r, \quad w=w_{\infty} .
$$

The constant $\omega$ is the angular velocity of the rotating disk in von Kármán's problem $(\kappa=0)$ and of the rotating fluid in Bödewadt's problem $(\kappa=1)$. The flow fields (26) lead to the energy equation

$$
T_{r r}+\frac{T_{r}}{r}=0
$$

with the general solution

$$
T=\alpha+\beta \log r .
$$

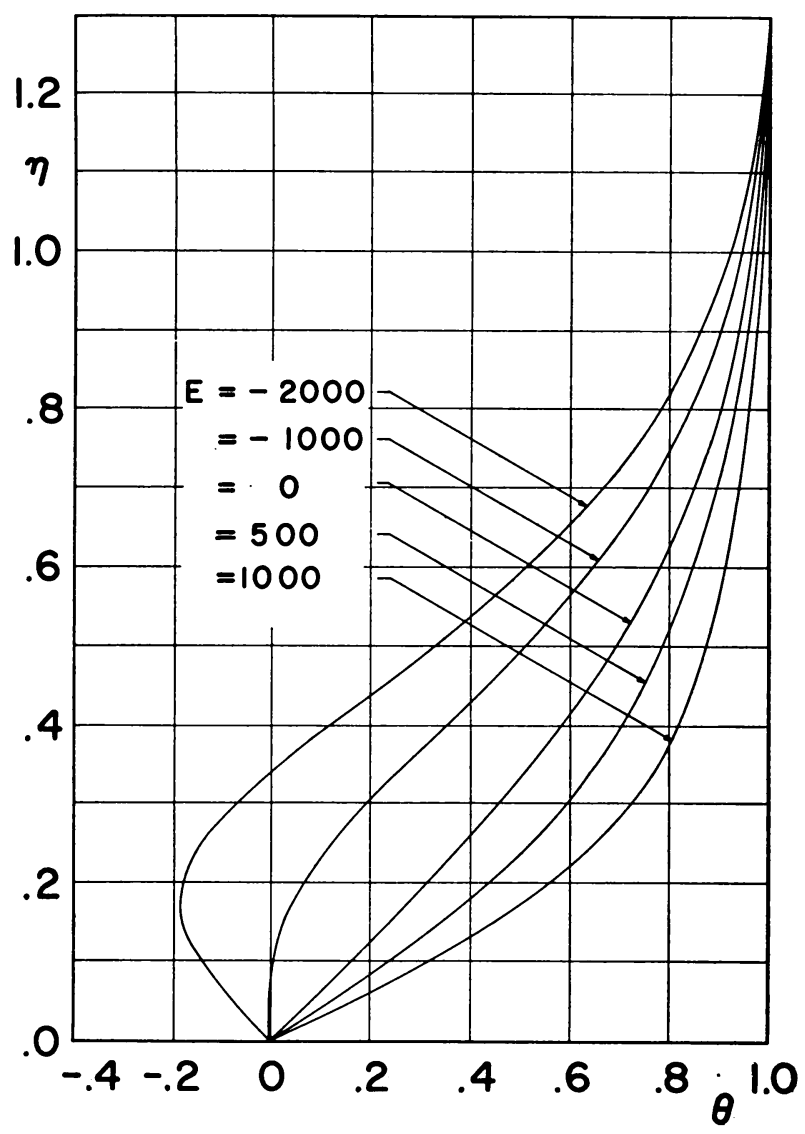

Fig. 8. The dimensionless temperature $\theta$ of Kármán flows vs. the dimensionless variable $\eta$ for $R=1, P_{r}=7$, and for different $E$. 
A comparison with Eq. (7) shows that no dissipation effects occur outside the boundary layer. This is due to the solid-body motion of the fluid for both the von Kármán and the Bödewadt problems.

For the following derivations no heat source is assumed at the axis of rotation $(\beta=0)$. Therefore, the temperature far away from the disk is a constant $\alpha=T_{\infty}$ at one's disposal. Assuming an isothermal disk one has the complete boundary conditions (see [2]):

$$
\begin{aligned}
& \left.\begin{array}{r}
z=0 \\
|r|<\infty
\end{array}\right\}: u=0, \quad v=(1-\kappa) \omega r, \quad w=0, \quad T=T_{w}, \\
& \left.\begin{array}{r}
z=\infty \\
|r| \leq \infty
\end{array}\right\}: u=0, \quad v=\kappa \omega r, \quad w=w_{\infty}, \quad T=T_{\infty} \text {, } \\
& \left.\begin{array}{c}
z>0 \\
|r| \rightarrow \infty
\end{array}\right\}: \frac{u}{\omega r} \rightarrow 0, \quad \frac{v}{\omega r} \rightarrow \kappa, \quad w \rightarrow w_{\infty}, \quad T \rightarrow T_{\infty} .
\end{aligned}
$$

In both problems no boundary conditions are prescribed at the two points $(r= \pm \infty$, $z=0)$. However, the strengths of these singularities are specified by the constant $w_{\infty}$ which is at one's disposal.

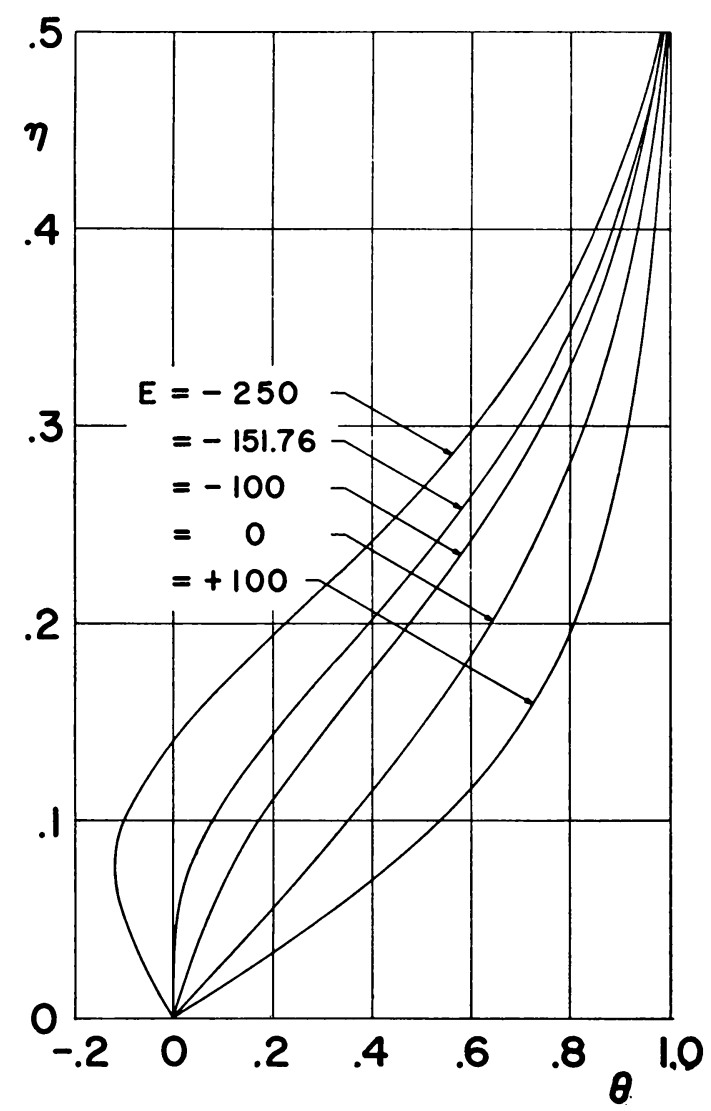

Fig. 9. The dimensionless temperature $\theta$ of Kármán flows vs. the dimensionless variable $\eta$ for $R=100, P_{r}=.7$, and for different $E$. 
The reduction of the Navier-Stokes equations was achieved in [2] by means of the transformation (11) and the "local boundary layer approximation of first order"

$$
\begin{gathered}
u=-\omega r g^{\prime}(\eta) \quad v=\omega r V(\eta), \quad w=2 \omega l g(\eta), \\
\frac{p}{\rho}=\frac{\omega^{2} r^{2}}{2} \kappa^{2}-2 \omega^{2} l^{2} H(\eta), \quad R=\frac{\omega}{\nu} l^{2}, \quad w_{\infty}=2 \omega l g_{\infty},
\end{gathered}
$$

where $l$ is the characteristic length defined as the geometric mean of the boundary layer thickness and twice its radius of curvature at the axis of rotation.

The corresponding approximation for the temperature $T$ is now introduced as

$$
\frac{T-T_{w}}{T_{\infty}-T_{w}}=\theta(\eta),
$$

and the energy equation (5) becomes

$$
\begin{gathered}
{\left[1+\eta^{2}\left(\frac{d \gamma}{d r}\right)^{2}\right] \theta^{\prime \prime}-\left[\eta\left(\frac{\gamma}{r} \frac{d \gamma}{d r}+\gamma \frac{d^{2} \gamma}{d r^{2}}-2\left(\frac{d \gamma}{d r}\right)^{2}\right\}+\frac{\omega \gamma P_{r}}{\nu}\left(2 l g+r \eta g^{\prime} \frac{d \gamma}{d r}\right)\right] \theta^{\prime}} \\
=-4 \frac{\omega^{2} \gamma^{2} P_{r}}{c\left(T_{\infty}-T_{w}\right)}\left[\left\{1+\frac{2 l^{2}}{\gamma^{2}}+\left(\frac{l \eta}{\gamma} \frac{d \gamma}{d r}\right)^{2}\right\} g^{\prime 2}-\left(1+\frac{l}{\gamma}\right) \frac{r \eta}{\gamma} g^{\prime} g^{\prime \prime} \frac{d \gamma}{d r}\right. \\
\left.+\frac{r^{2}}{4 \gamma^{2}}\left\{1+2 \eta^{2}\left(\frac{d \gamma}{d r}\right)^{2}\right\} g^{\prime \prime 2}+\frac{r^{2}}{4 \gamma^{2}}\left\{1+\eta^{2}\left(\frac{d \gamma}{d r}\right)^{2}\right\} V^{\prime 2}\right] .
\end{gathered}
$$

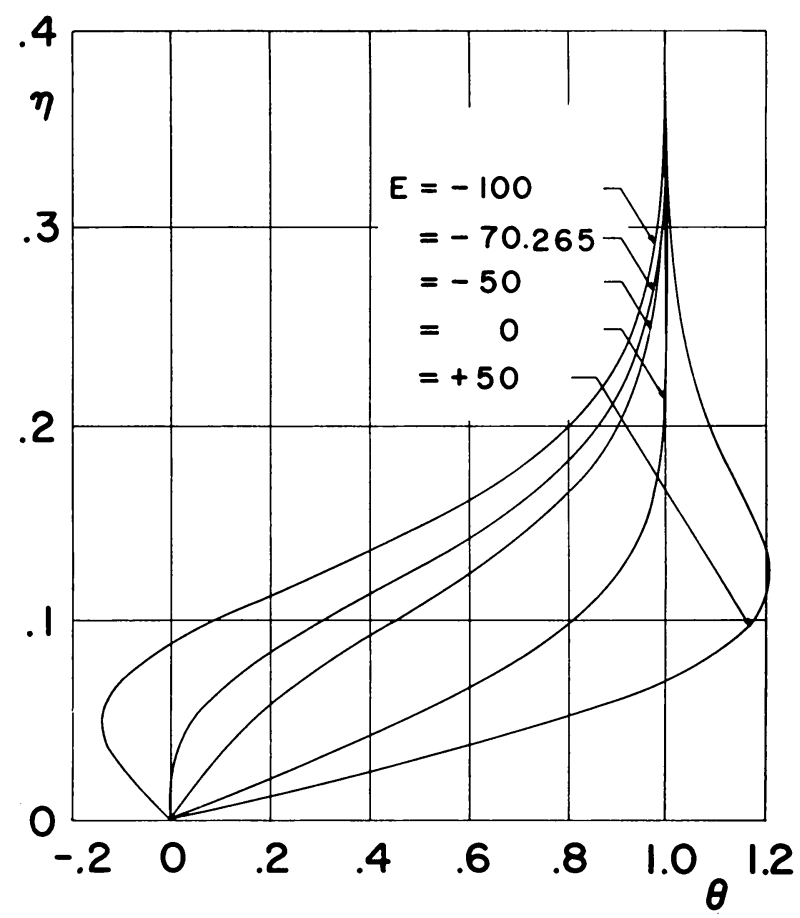

Fig. 10. The dimensionless temperature $\theta$ of Kármán flows vs. the dimensionless variable $\eta$ for $R=100, P_{r}=7$, and for different $E$. 
The limiting line of the boundary layer has been found in [2] as

$$
z=\sigma \gamma(r)=\sigma\left(l-\frac{1}{l} r^{2}+\cdots\right) .
$$

For $r \rightarrow 0$ Eq. (34) reduced to

if the constant

$$
\theta^{\prime \prime}+2\left(2 \eta-P_{r} R g\right) \theta^{\prime}+12 P_{r} E g^{\prime 2}=0
$$

$$
E=\frac{\omega^{2} l^{2}}{c\left(T_{\infty}-T_{w}\right)}
$$

is interpreted as an Eckert number. The boundary conditions are

$$
\begin{aligned}
\eta & =0: & \theta & =0, \\
\eta & =\infty: & \theta & =1 .
\end{aligned}
$$

In contrast to the energy equation of the vortex flow, which has been described in the previous section, only one Eckert number occurs. This is due to the restriction $\beta=0$, which excludes a heat source at the axis $r=0$.

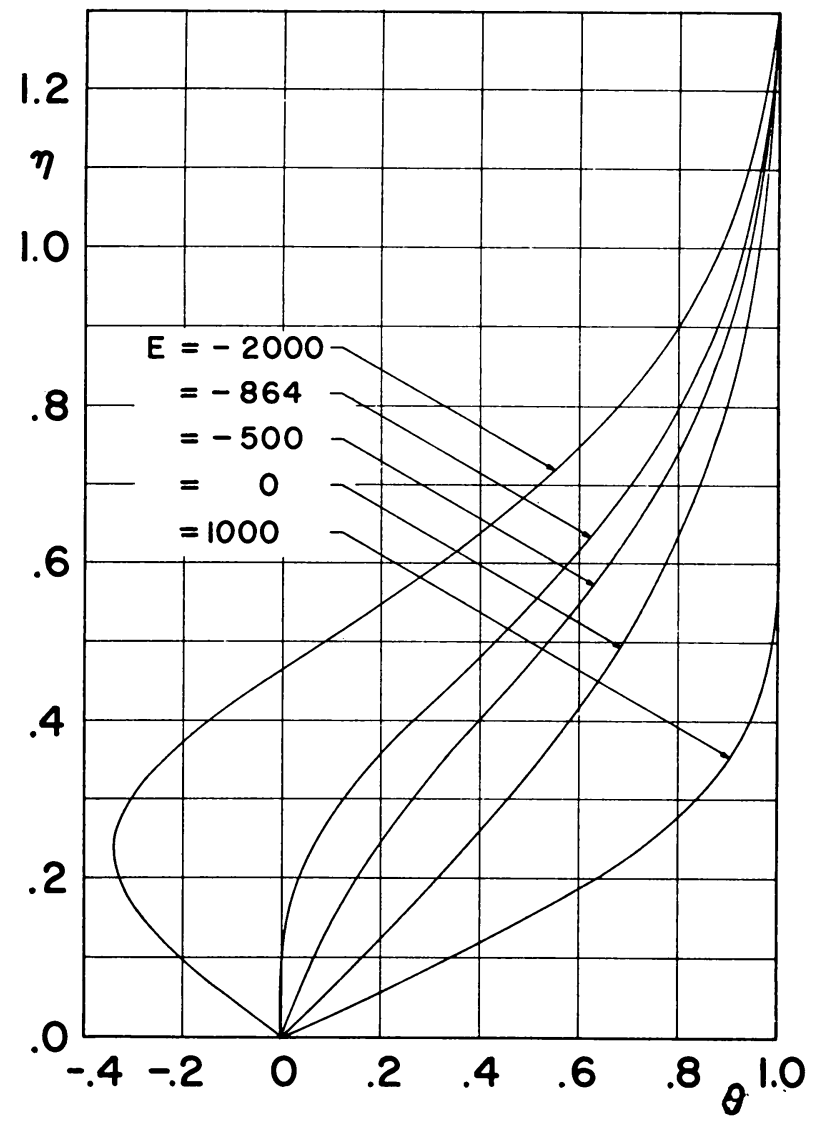

FIg. 11. The dimensionless temperature $\theta$ of Bödewadt flows vs. the dimensionless variable $\eta$ for $R=1, P_{r}=.7$, and for different $E$. 
Since $g$ is a known function, which is listed in [2], Eq. (36) is a linear differential equation for $\theta$, the solution of which is

$$
\begin{aligned}
& \theta(\eta)=\int_{0}^{\eta} \exp 2\left\{-t^{2}+P_{r} R \int_{0}^{t} g(s) d s\right\}[ \lambda-12 P_{r} E \int_{0}^{t} g^{\prime 2}(s) \\
&\left.\cdot \exp 2\left\{s^{2}-P_{r} R \int_{0}^{s} g(q) d q\right\} d s\right] d t
\end{aligned}
$$

where

$$
\begin{aligned}
\lambda=\left[1+12 P_{r} E \int_{0}^{\infty} \exp 2\left\{-\eta^{2}+\right.\right. & \left.P_{r} R \int_{0}^{\eta} g(t) d t\right\} \int_{0}^{\eta} g^{\prime 2}(t) \\
& \left.\cdot \exp 2\left\{t^{2}-P_{r} R \int_{0}^{t} g(s) d s\right\} d t d \eta\right] \\
& \cdot\left[\int_{0}^{\eta} \exp 2\left\{-\eta^{2}+P_{r} R \int_{0}^{\eta} g(t) d t\right\} d \eta\right]^{-1}
\end{aligned}
$$

according to the boundary conditions (38) and (39). Numerical results are presented in Sect. 6.

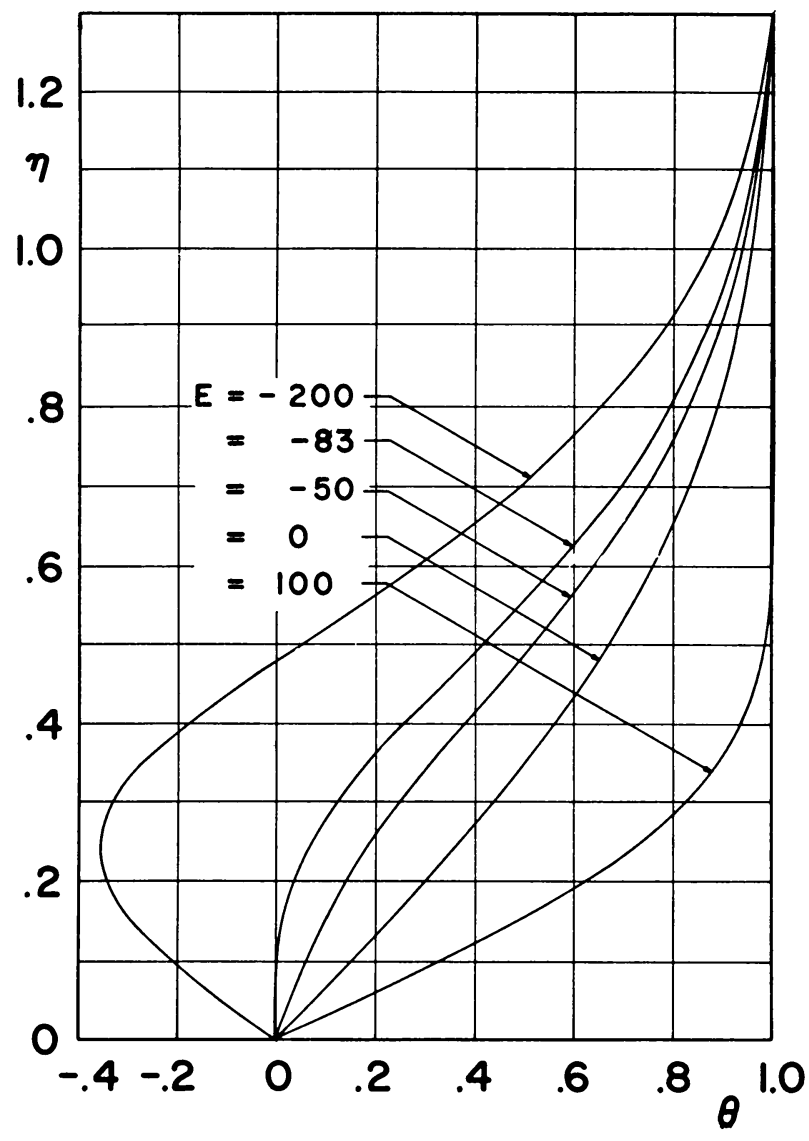

Fig. 12. The dimensionless temperature $\theta$ of Bödewadt flows vs. the dimensionless variable $\eta$ for $R=1, P_{r}=7$, and for different $E$. 
5. Characteristics of temperature fields of vortex flows. The heat transfer from a flat surface by vortex flows, which is described in Sect. 3, has been calculated for the Reynolds numbers 1 and 100, for the Prandtl numbers 0.7 (air) and 7 (water), and for various Eckert numbers $E_{0}$ and $E_{1}$. The temperature profiles are plotted in Figs. 2 through 6, and their corresponding data are tabulated in [9]. Before the discussion of these graphs a brief comment is given on the physical meaning of the parameters $E_{0}$ and $E_{1}$, and the slopes of the profiles at the surface.

The Eckert number $E_{0}$ is a measure of the dissipative heat production over the conductive and convective heat transfer within the fluid. Vanishing $E_{\text {J }}$ is equivalent to negligible friction effects, whereas increasing absolute values of $E_{0}$ lead to larger frictional influence and finally to complete domination of friction over conductive and convective heat effects. The sign of $E_{0}$ determines according to its definition (17), whether the surface is cooler or warmer than the fluid at infinity at $r_{0}$.

The parameter $E_{1}$ represents essentially the strength of a heat source in the core of the vortex. The value $1 / E_{1}=0$ states that no heat other than a dissipative one is produced in the core. Positive values of $E_{1}$ correspond to a heat source in the core, negative values are equivalent to a heat sink.

The slope of the temperature profile at the surface determines the heat flux from the surface to the fluid. Therefore, the dimensionless temperature gradient at the surface may be interpreted as the Nusselt number $N$ (see [10])

$$
N=\left(\frac{d \theta}{d \eta}\right)_{\eta=0} .
$$

The direction of the heat flux depends on the sign of $\mathrm{E}_{0}$. For positive $E_{0}$ positive Nusselt numbers correspond to a heat flux from the surface to the fluid, negative Nusselt numbers correspond to heat flowing from the fluid to the surface. For negative $E_{0}$ these relations are reversed.

In Fig. 2 the temperature profiles $\theta(\eta)$ are plotted for $R=1, P_{r}=.7,1 / E_{1}=0$, and for various $E_{0}$. For $E_{0}=0$ the energy equation (16) becomes integrable by the error function, which is independent of the other parameters $R, P_{r}$, and $E_{1}$. The heat flux for this important special case is $N=1.128$. Increasing negative values of $E_{0}$-the surface is in this case warmer than the fluid-enhance the friction effects in such a way, that the lower part of the boundary layer is heated more and more. Hence, the temperature difference between surface and fluid diminishes, and the heat flux from the surface (i.e., the Nusselt number) becomes smaller. For $E_{0} \approx-2$ the heat flux is zero. Beyond this value the heat flux reverses. The boundary layer is warmer than the surface because of the frictional heat. This phenomenon is well known for the Couette flow (see [10]). Increasing positive values of $E_{0}$ - the surface is cooler than the fluidcorrespond also to a heating of the boundary layer, but because of the increasing temperature difference between surface and fluid the heat flux to the surface is enhanced. From $E_{0} \approx 50$ on the temperature within the boundary layer exceeds the temperature outside the boundary layer. This effect is also well known for the heat transfer from a flat plate (see [10]). Corresponding statements can be made for $R=1, P_{r}=7$ (Fig. 4), for $R=100, P_{r}=.7$ (Fig. 5), and for $R=100, P_{r}=7$ (Fig. 6). The Nusselt numbers for various $R, P_{r}, E_{0}$, and $E_{1}$ are listed in Table 1 .

In Fig. 3 the parameter $E_{1}$ has been varied for fixed values $R=1, P_{r}=.7$, and $E_{0}=5$. Since the surface is assumed to be cooler than the fluid $\left(E_{0}>0\right)$ decreasing 
negative values of $E_{1}$-the core is cooled too-cause a domination of frictional heat over conduction and convection. This effect may be compared with that of increasing $E_{0}$ (see Fig. 2). The temperature within the boundary layer exceeds the temperature outside the boundary layer for $1 / E_{1}<-5$, whereas the heat flux increases only slightly. For decreasing positive values of $E_{1}$-the core is heated-the effects are reversed.

The Prandtl number is inversely proportional to the conductivity $k$. The increase of the Prandtl number diminishes, therefore, the heat conduction and causes an effect similar to that of increasing Eckert number $E_{0}$. This is expected according to the energy equation (16) and is confirmed by Figs. 4 and 6, which must be compared with Figs. 2 and 5 , respectively. Because of the stronger frictional effect the absolute values of the Nusselt numbers are higher for $P_{r}=7$ than for $P_{r}=.7$ (see Table 1).

Increasing Reynolds numbers enlarge frictional effects. This can be seen in Table 1 and by comparing Figs. 2 and 5, and Figs. 4 and 6.

Table 2: Nusselt Numbers of Von Kármán Flows for Various $R, P_{r}$, and $E$.

1. $R=1, \quad P_{r}=.7$

\begin{tabular}{l|rrrrrrr}
$E$ & -20000 & -10000 & -1000 & 0 & 1000 & 5000 & 10000 \\
\hline$N$ & -1.61 & 0.004 & 1.45 & 1.61 & 1.77 & 2.42 & 3.22
\end{tabular}

2. $R=1, \quad P_{r}=7$

\begin{tabular}{l|rrrrrrr}
$E$ & -2000 & -1000 & -100 & 0 & 100 & 500 & 1000 \\
\hline$N$ & -1.58 & 0.022 & 1.47 & 1.63 & 1.79 & 2.43 & 3.23
\end{tabular}

3. $R=100, P_{r}=.7$

\begin{tabular}{l|rrrrr}
$E$ & -250 & -151.76 & -100 & 0 & +100 \\
\hline$N$ & -2.35 & 0.000 & 1.24 & 3.63 & 6.03
\end{tabular}

4. $R=100, \quad P_{r}=7$

\begin{tabular}{l|rrrrr}
$E$ & -100 & -70.265 & -50 & 0 & +50 \\
\hline$N$ & -4.11 & 0.000 & 2.80 & 9.72 & 16.6
\end{tabular}

5. $E=0, P_{r}=.7$

\begin{tabular}{l|rrrrr}
$R$ & .1 & 1 & 10 & 50 & 100 \\
\hline$N$ & 1.6108 & 1.61 & 1.74 & 2.63 & 3.63
\end{tabular}

6. $E=0, \quad P_{r}=7$

\begin{tabular}{l|rrrrr}
$R$ & .1 & 1 & 10 & 50 & 100 \\
\hline$N$ & 1.6109 & 1.63 & 2.71 & 6.7 & 9.7
\end{tabular}

7. $E=0, \quad P_{r}=70$

\begin{tabular}{l|rrrrr}
$R$ & .1 & 1 & 10 & 50 & 100 \\
\hline$N$ & 1.6124 & 1.77 & 6.4 & 16 & 23
\end{tabular}


Table 3: Nusselt Numbers of Bödewadt Flows for $R=1$ and for Various $P_{r}$ and $E$

1. $P_{r}=.7$

\begin{tabular}{l|rrrrr}
$E$ & -2000 & -864 & -500 & 0 & 1000 \\
\hline$N$ & -2.11 & -.001 & +.676 & 1.61 & 3.46
\end{tabular}

2. $P_{r}=7$

\begin{tabular}{l|rrrrr}
$E$ & -200 & -83 & -50 & 0 & 100 \\
\hline$N$ & -2.20 & -.001 & +.619 & 1.56 & 3.44
\end{tabular}

Finally, it may be mentioned that in all cases the thermal boundary layers have nearly the same thickness as the associated momentum boundary layers.

6. Characteristics of temperature fields of von Kármán-Bödewadt flows. The temperature profiles of von Kármán-Bödewadt flows have been calculated by Eq. (40) for the Prandtl numbers .7 and 7, for various Eckert numbers $E$, and for the Reynolds numbers 1 and 100 for Kármán flows, the Reynolds number 1 for Bödewadt flows. The data are tabulated in [9] and plotted in Figs. 7 through 12. The physical meaning of $E$ corresponds to that of $E_{0}$ in the preceding section, so that all statements made about the influence of $E_{0}$ apply also to the Figs. 7 through 12. In Tables 2 and 3 the Nusselt numbers are listed.

The thermal boundary layers of the flows of von Kármán and Bödewadt differ in their dependence on the Eckert numbers, which is stronger for Bödewadt flows than for von Kármán flows (Figs. 7, 8, 11, and 12). This can be seen immediately from Eq. (65) and the fact that the radial velocity components are larger for Bödewadt flows than for von Kármán flows. In the special case $E=0$, the Nusselt numbers for Bödewadt flows decrease slightly with larger Prandtl numbers in contrast to von Kármán flows.

Negligible dissipation $(E=0)$ is of particular interest since theoretical and experimental data of von Kármán flows exist in literature. In Fig. 13 the Nusselt numbers

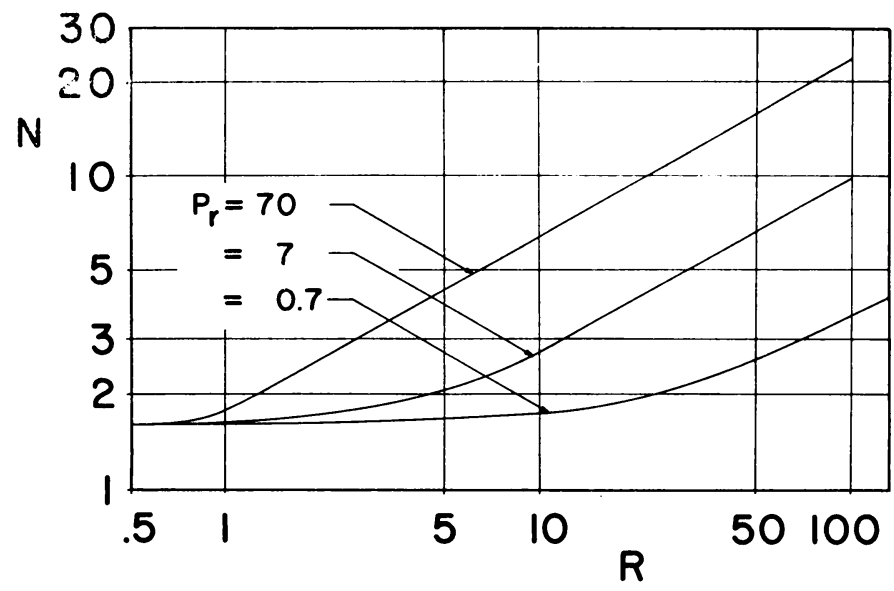

Fig. 13. The Nusselt number $N$ vs. the Reynolds number $R$ of von Kármán flows for $E=0$ and for three different Prandtl numbers. 
are plotted versus the Reynolds numbers for three different Prandtl numbers. In order to compare the experimental data by Cobb and Saunders [8] with the new theoretical results, the characteristic length $l$ of the mathematical model of infinite dimensions must be adjusted to the characteristic length of the dynamically similar apparatus used by Cobb and Saunders. This can be achieved by the following approximation. According to their definitions the Reynolds numbers and Nusselt numbers of both models satisfy the relations

$$
\frac{N}{N_{e}}=\frac{l}{l_{e}}, \quad \frac{R}{R_{e}}=\frac{l^{2}}{l_{e}^{2}}
$$

where the subscript $e$ designates experimental values. Thus, if an experimental pair $\left(R_{e 1}, N_{e 1}\right)$ is specified, one must seek the corresponding pair $\left(R_{1}, N_{1}\right)$ which satisfies the equation

$$
\frac{R_{1}}{R_{e 1}}=\frac{N_{1}^{2}}{N_{e 1}^{2}}
$$

In the present case the ratio $l / l_{e}$ was found to be approximately 31 .

In Fig. 14 the experimental data by Cobb and Saunders are plotted together with the new adjusted curve for $P_{r}=0.7$ and with Wagner's solution [3], which is based on von Kármán integral. The agreement between theory and experiment is satisfactory. It is interesting that similar to the results obtained in [2] Wagner's straight line appears as an asymptote of the new and experimental values (the small displacement of Wagner's straight line can be corrected after [8] and [4]). Exactly the same asymptotic agreement can be observed between the new results and those presented by Sparrow and Gregg in [5] for the Prandtl numbers .7, 7, and 70. Thus, the supposition is confirmed that von Kármán's rotating disk solution is an asymptotic integral.

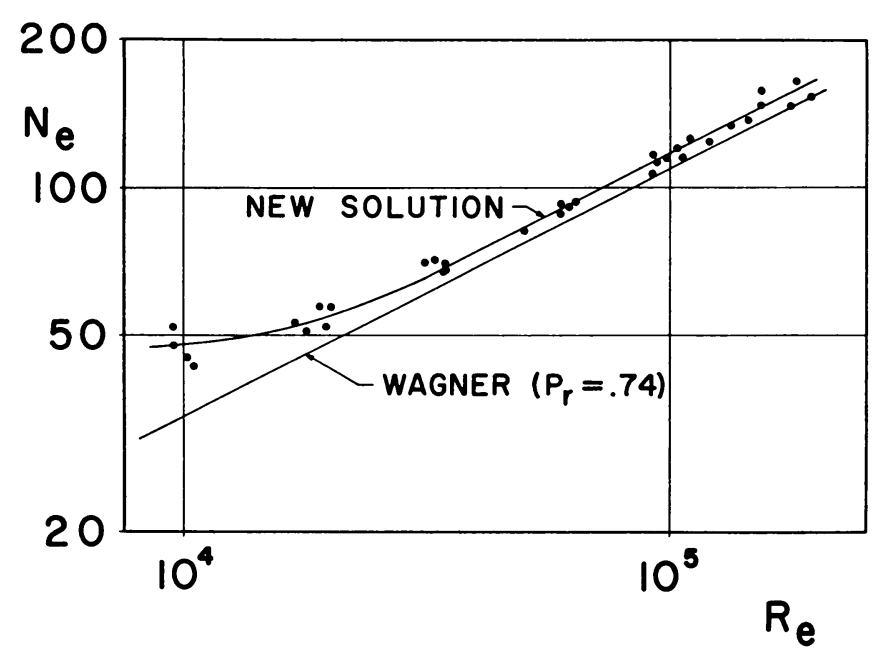

Fig. 14. Experimental data by Cobb and Saunders [8] for the laminar flow region. Nusselt number and Reynolds number are defined as

$$
N_{e}=h r_{0} /\left(T_{w}-T_{\infty}\right) \quad R_{e}=\omega r_{0}^{2} / \nu
$$

where $h=$ mean heat transfer coefficient for the whole disk

$r_{0}=$ radius of the disk. 
Acknowledgment. The authors wish to extend their thanks to Dr. C. J. Cohen for many critical and stimulating discussions.

\section{REFERENCES}

1. E. W. Schwiderski and H. J. Lugt, Vortex flows normal to a flat surface, Tellus 15 (1963) 241

2. E. W. Schwiderski and H. J. Lugt, Rotating flows of von Karman and Bödewadt, Phys. of Fluids 7 (1964) 867

3. C. Wagner, Heat transfer from a rotating disk to ambient air, Journ. Appl. Phys. 19 (1948) 837

4. K. Millsaps and K. Pohlhausen, Heat transfer by laminar flow from a rotating plate, Journ. Aer. Sci. 19 (1952) 120

5. E. M. Sparrow and J. L. Gregg, Heat transfer from a rotating disk to fluids of any Prandtl number, Trans. ASME Series C 81 (1959) 249

6. Th. von Kármán, Über laminare und turbulente Reibung, ZAMM 1 (1921) 244

7. R. L. Young, Heat transfer from a rotating plate, Trans. ASME 78 (1956) 1163

8. E. C. Cobb and O. A. Saunders, Heat transfer from a rotating disk, Proc. Roy. Soc. Series A 236 (1956) 343

9. H. J. Lugt and E. W. Schwiderski, Heat transfer from a flat surface by rotating flows, U. S. Naval Weapons Laboratory Report No. 1875, 1963

10. H. Schlichting, Boundary layer theory, Fourth Edition, 1960 